REV EXP MED 2020;6(4).

\title{
COVID-19 y la investigación médica: ¿El fin justifica los medios?
}

\section{COVID-19 and medical research: Does the end justify the means?}

\author{
Víctor Díaz-Silva ${ }^{1, a}$
}

Después que la Organización Mundial de Salud emitiera la alerta sobre la pandemia por COVID-19, el número de investigaciones relacionadas a esta enfermedad y las publicaciones de artículos de investigación se han incrementado notablemente (1). Los denominados artículos "originales" o papers que buscaban respuestas a los mecanismos de propagación de la infección, sobre los tratamientos o respecto a los factores pronósticos se publicaban día a día y algunos con resultados contradictorios o con metodologías cuestionables.

La investigación científica tiene parámetros de rigor establecidos y aceptados internacionalmente, pero los últimos meses las editoriales fomentaron la publicación de investigaciones "acelerando" el proceso de revisión. La rigurosidad de los criterios de investigación se vio afectada, tan es así que organismos responsables de revisión de la evidencia como el Instituto de Evaluación de Tecnologías en Salud e Investigación (IETSI) encontraban deficiencias metodológicas en las investigaciones sobre los tratamientos contra la COVID-19 (2). Algunos medicamentos como la ivermectina, hidroxicloroquina, tocilizumab, remdesivir, entre otros, fueron inicialmente recomendados o considerados en las guías de manejo contra la COVID-19 sin mayor evidencia clínica.

Las buenas prácticas en investigación deben predominar sobre cualquier circunstancia, y no es lo que se ha observado en tiempos de la COVID-19 donde al parecer ha primado la búsqueda de resultados antes que la rigurosidad científica. Es ético respetar todos los criterios del rigor científico ya que una investigación deficiente o inconclusa puede ser perjudicial para la salud pública. Un ejemplo de ello fue la administración de ivermectina o de hidroxicloroquina, medicamentos que tenían actividad in-vitro frente al virus, pero no comprobada clínicamente, y fueron utilizados durante los primeros días de la pandemia ${ }^{(3,4)}$, lo que conllevó, junto a otros factores, a una elevada tasa de mortalidad en muchas localidades.

Cabe mencionar que la pandemia causó, además, el abandono en la investigación de otras enfermedades crónicas prevalentes. Se dejaron de lado investigaciones en enfermedades infecciosas o metabólicas o crónicas; sin embargo, esta situación se está corrigiendo al investigar el comportamiento en pacientes afectados por COVID-19 que eran portadores de una enfermedad crónica.

Finalmente, los avances y errores en la investigación sobre la COVID-19 han demostrado que la investigación básica y clínica es necesaria, que se debe respetar el rigor científico y ético en toda circunstancia y que no necesariamente los resultados de experimentos in-vitro o de pequeños grupos son extrapolables a la práctica clínica individual o de salud pública; esto último es una aseveración que ya se conocía pero que pensamos podía ser diferente en tiempos de pandemia.

\footnotetext{
1. Hospital Regional Lambayeque. Lambayeque, Perú.

a. Magíster en Medicina. Especialista en Hematología Clínica.

ORCID: 0000-0002-4595-6458
} 


\section{Conflictos de interés: El autor declara que no existe} conflicto de interés.

\section{Fuentes de financiamiento: Autofinanciado}

\section{REFERENCIAS BIBLIOGRÁFICAS}

1. PubMed [Internet]. Bethesda (MD): National Center for Biotechnology Information (US); 1996- [citado 5 dic 2020]. Disponible en: http://www. ncbi.nlm.nih.gov/pubmed/

2. Instituto de Evaluación de Tecnologías en Salud e Investigación [Internet]. Perú: Essalud; c2020. [actualizado 08 set 2020; citado 7 dic 2020]. Reportes breves COVID-19. Subdirección de evaluación de productos farmacéuticos y otras tecnologías sanitarias; [aprox. 1 pag], Disponible en: http://www.essalud.gob.pe/ietsi/eval_prod_farm_otros_covid19_report.html

3. Bendezu-Quispe G, Rodríguez-Zúñiga MJM, Roman YM, Mori-Llontop LM, Peralta V, Fiestas F. Agentes potencialmente terapéuticos contra el SARS-CoV-2: revisión rápida de la evidencia. Rev Peru Med Exp Salud Publica. 2020;37(2):320-6. doi: https://doi.org/10.17843/rpmesp.2020.372.5409

4. Ministerio de Salud del Perú. "Resolución Ministerial N 139-2020-MINSA" [Internet]. Lima; 2020 [citado 05 dic 2020]. Disponible en: https:// www.gob.pe/institucion/minsa/normas-legales/465962-139-2020-minsa 